\title{
CECT FEATURES OF RETROPERITONEAL FIBROSIS WITH BILATERAL OBSTRUCTIVE UROPATHY SECONDARY TO ANEURYSM AND DISSECTION OF ABDOMINAL AORTA
}

Abhang V. Apte ${ }^{1}$, Vinod Y. Attarde ${ }^{2}$, Parag V. Patil ${ }^{3}$, Sarvesh Patil ${ }^{4}$

\section{HOW TO CITE THIS ARTICLE:}

Abhang V. Apte, Vinod Y. Attarde, Parag V. Patil, Sarvesh Patil. "CECT Features of Retroperitoneal Fibrosis with Bilateral Obstructive Uropathy Secondary to Aneurysm and Dissection of Abdominal Aorta". Journal of Evolution of Medical and Dental Sciences 2014; Vol. 3, Issue 10, March 10; Page: 2586-2589,

DOI: $10.14260 /$ jemds/2014/2177

ABSRACT: Retroperitoneal fibrosis is one of uncommon cause of obstructive uropathy. Aortic dissection without rupture is still uncommon cause of retroperitoneal fibrosis. We present unusual case of aortic aneurysm with dissection without its acute rupture causing retroperitoneal fibrosis with its contrast computed tomography features. We report imaging features of computed tomography of abdominal aortic dissection complicated by retroperitoneal fibrosis with bilateral ureteral obstruction. The patient underwent successful bilateral transurethral ureteral stenting to relieve acute obstruction and started on corticosteroid treatment.

KEYWORDS: Retroperitoneal fibrosis, obstructive uropathy, abdominal aortic dissection, computed tomography.

CASE: A 40-year-old female complains of anuria and vague abdominal pain came to surgery department. USG features were suggestive of bilateral hydronephrosis and proximal hydroureter with dilatation of aorta. Her serum BUN and creatinine levels were slightly raised.

She underwent non contrast CT to look for cause of renal obstruction. Non contrast CT shows dilatation of bilateral pelvicalyceal systems with proximal hydroureters upto L3 -L4 vertebral level. Abdominal aorta seen dilated at this level with atheromatous changes in the form of vessel wall calcification. Minimal hypodensity seen at periaortic region adjacent to mid ureter. IV contrast study was done to delineate aortic pathology better. CECT study shows an abdominal aortic aneurysm (3 $\mathrm{cm}$ in diameter) distal to the origin of the renal arteries and was encircled by a very small amount of nonenhancing hypodense soft tissue density i.e., fibrotic tissue .There was short segment dissection in abdominal aorta. Bilateral hydronephrosis with hydroureter are seen up to this level. The interpretation of these findings is minimal RPF with aortic aneurysm and dissection. Patient was referred to urology department for further management.

INTRODUCTION: Although extrinsic ureteral stenosis is reported in up to $71 \%$ of patients referred for abdominal aneurysms ${ }^{1}$ ureteral obstruction secondary to perianeurysmal fibrosis is uncommon. ${ }^{2}$ The cause of such fibrosis is still unknown. Periureteral extension of the inflammatory disease that has affected the aorta and mere coincidence (the coexistence of idiopathic retroperitoneal fibrosis and an abdominal aneurysm) are the 2 main pathophysiologic mechanisms that have been proposed. ${ }^{3,4}$

Retroperitoneal fibrosis (RPF) is defined as an inflammatory and fibrotic mass that develops in the retroperitoneum.Albarran ${ }^{5}$ first described the disease in 1905 , but it was not until Ormond 6 reported cases of RPF in 1948 that it became a recognized clinical entity. The disease became 
acknowledged as RPF in the 1960s, and this remains the current preferred term. A variety of terms have been used to describe the disorder, including Ormond's disease, periureteritis fibrosa, periureteritis plastica, chronic periureteritis, sclerosing retroperitoneal granuloma, and fibrous retroperitonitis. In most patients (approximately 68\%), no etiologic factor is found. Therefore, the term idiopathic RFP is used. Evidence suggests that RPF is an autoimmune response to an insoluble lipid called ceroid that has leaked through a thinned arterial wall from atheromatous plaques. ${ }^{7,8}$

Other implicated causes include drugs, abdominal aortic aneurysm, ureteric renal injury, infection, retroperitoneal malignancy, post irradiation therapy, chemotherapy and hemilaminectomy, hypothyroidism and carcinoid tumor.

More than $90 \%$ patients presents gradually as acute renal colic. Constitutional symptoms like malaise, anorexia, fever, weight loss, neurovascular compromise can be present. Venous complications are may be due to IVC involvement like lower extremity edema, phlebitis, DVT. Arterial complications are may be due major arterial involvement like Claudication, mesenteric ischemia. Symptoms of renal insufficiency or failure from obstructive uropathy at the ureteral level.

Previously plain radiograph, intravenous urography and retroperitoneal pyelography have been used as imaging modalities. Computed tomography scanning and magnetic resonance imaging provide superior delineation of the extent of the masses of RPF. ${ }^{9-12}$

Isotope renography is useful in the serial assessment of renal function. Gallium scintigraphy may demonstrate increased uptake, depending on the activity of inflammation. The use of fluorodeoxy glucose positron emission tomography (FDG-PET) in differentiating benign from malignant RPF is promising. ${ }^{13-15}$

With CT and MRI, the anatomical extent and involvement of other structures can be shown in more detail. The symmetrical distribution and geometric shape are highly suggestive of RPF

RPF can be seen in detail on CT scans. The exact etiology cannot be identified, but it can be suggested by excluding other identifiable causes.

On CT scans, RPF may appear as a rind of soft tissue around the aorta and inferior vena cava extending between the renal hilum and sacral promontory. Laterally, RPF spreads to involve the ureters, causing varying degrees of obstruction.

The fat plane between the mass and the psoas muscle may be obliterated. The mass tends not to displace the aorta anteriorly. The attenuation value of the mass is similar to that of muscle and shows variable degrees of enhancement depending on the stage of the disease.

Certain CT features can help in differentiating benign masses from malignant masses. The mass in RPF may be bulky but not as massive as neoplastic lesions; however, some exceptions exist. The presence of enlarged mesenteric nodes and displacement of the aorta from the spine by the periaortic mass favors malignancy, although some displacement can occur in RPF. Unlike RPF, most retroperitoneal neoplasms displace the ureters laterally. RPF does not produce local bone destruction. The CT value and contrast enhancement have no role in distinguishing benign masses from malignant masses.

In conclusion, abdominal aortic dissection with aneurysm should be considered as a possible cause of ureteric obstruction. CT is the imaging modality used to diagnose RPF and to perform followup studies. CT scans help in assessing the extent of disease and in studying the effects on other organs. 


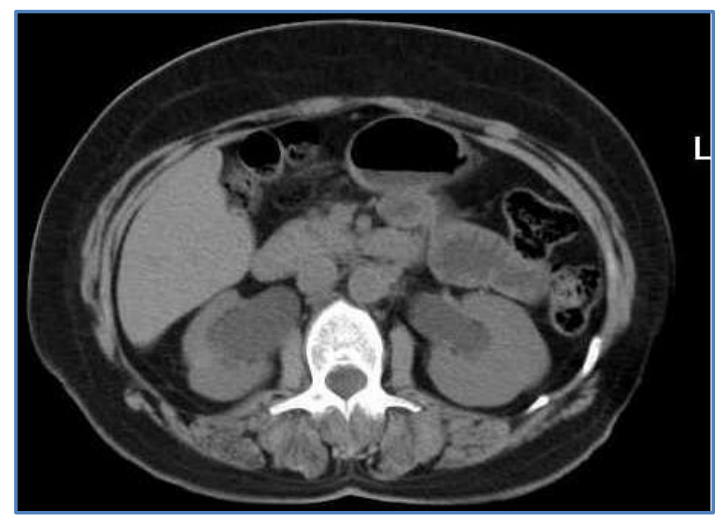

Fig. 1: Non-contrast CT axial image shows bilateral mild hydronephrosis with proximal hydroureter

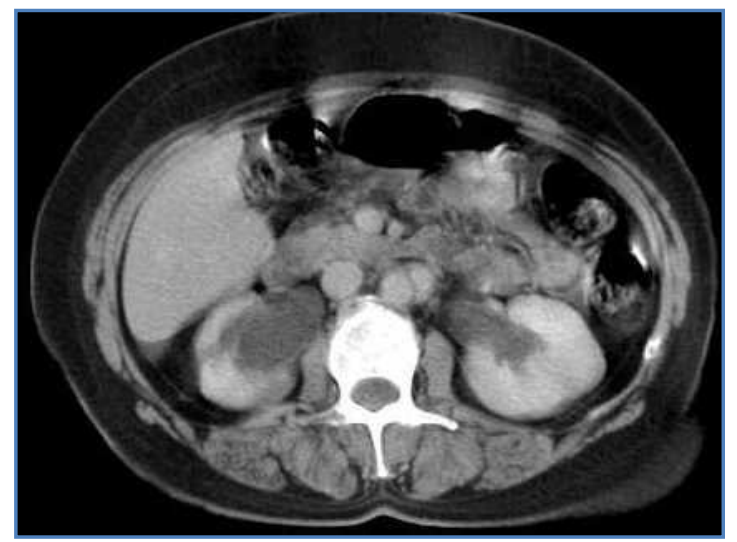

Fig. 3: Contrast CT axial image shows dilated aorta with aortic dissection

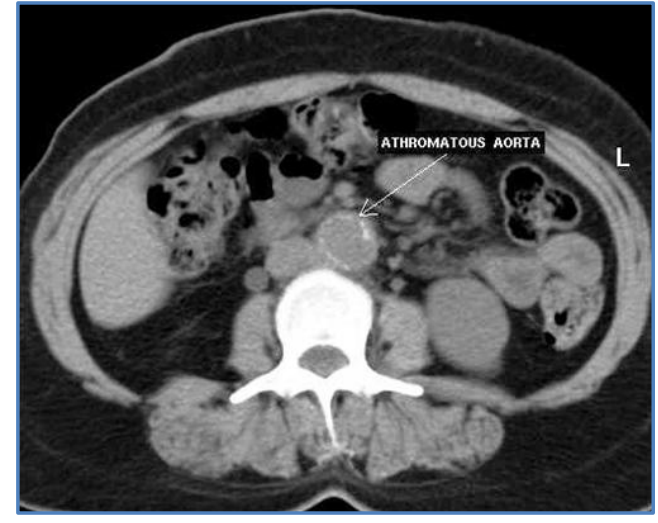

Fig. 2: Non-contrast CT axial image shows dilated aorta with atheromatous calcified plaque

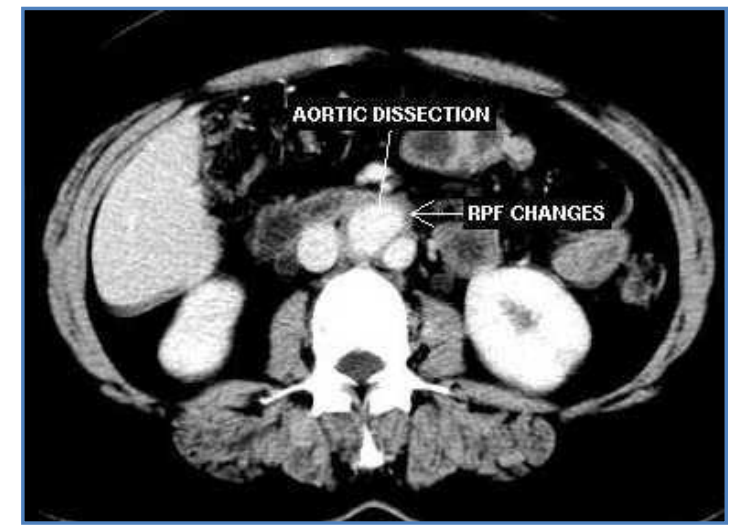

Fig. 4: Contrast CT axial image shows aortic dissection with minimal periaortic soft tissue

\section{REFERENCES:}

1. Hodgson KJ, Webster DJ. Abdominal aortic aneurysm causing duodenal and ureteric obstruction. J Vasc Surg 1986; 3:364-8.

2. Loughlin K, Kearney G, Helfrich W, Carey R. Ureteral obstruction secondary to perianeurysmal fibrosis. Urology 1984; 24:332-6.

3. Downs AR, Lye CR. Inflammatory abdominal aortic aneurysm. Can JSurg 1986; 29:50-3.

4. Nishino A, Kawaguchi K, Kakuma K. Bilateral ureteral obstruction by periarterial fibrotic reaction in aortoiliac arteriosclerosis: report of a case .Hinyokika Kiyo 1986; 32:865-70.

5. Albarran J. Retention rénale par periurétérité. Libérationexterne de l'uretère. 9. Association françaised'urologie; 1905:511.

6. Ormond JK. Bilateral ureteral obstruction due to envelopment and compression by an inflammatory retroperitoneal process. Journal of Urology, Baltimore 1948; 59:1072-1079.

7. Mitchinson MJ. Insoluble lipids in human atherosclerotic plaques. Atherosclerosis Oct 1982; 45(1):11-5. 
8. Parums DV, Brown DL, Mitchinson MJ. Serum antibodies to oxidized low-density lipoprotein and ceroid in chronic periaortitis. Arch Pathol Lab Med Apr 1990; 114(4):383-7.

9. Dixon AK, Mitchinson MJ, Sherwood T. Computed tomographic observations in periaortitis: a hypothesis. ClinRadiol Jan 1984; 35(1):39-42.

10. Ayuso JR, Garcia-Criado A, Caralt TM, et al. Atypical retroperitoneal fibrosis: MRI findings. EurRadiol 1999; 9 (5):937-9.

11. Ozgen A, Cila A. Renal involvement in multifocal fibrosclerosis: CT and MRI. J Comput Assist Tomogr Nov-Dec 1999; 23(6):937-8.

12. Elsayes KM, Staveteig PT, Narra VR, Chen ZM, Moustafa YL, Brown J. Retroperitoneal masses: magnetic resonance imaging findings with pathologic correlation. Curr Probl Diagn Radiol. MayJun 2007; 36(3):97-106.

13. Hillebrand M, Gerstenberg E, Kuntz RM, et al. [67Ga scintigraphy in retroperitoneal fibrosis (RPF): indications, application and clinical relevance--report of five patients]. Nuklearmedizin. Feb 1996; 35(1):25-30.

14. Strauss LG. Fluorine-18 deoxyglucose and false-positive results: a major problem in the diagnostics of oncological patients. Eur J Nucl Med. Oct 1996; 23(10):1409-15.

15. Jansen I, Hendriksz TR, Han SH, Huiskes AW, van Bommel EF. (18) F-fluorodeoxyglucose position emission tomography (FDG-PET) for monitoring disease activity and treatment response in idiopathic retroperitoneal fibrosis. Eur J Intern Med. Jun 2010; 21(3):216-21.

\section{AUTHORS:}

1. Abhang V. Apte

2. Vinod Y. Attarde

3. Parag V. Patil

4. Sarvesh Patil

\section{PARTICULARS OF CONTRIBUTORS:}

1. Associate Professor, Department of Radiology, MGM Medical College, Aurangabad.

2. Consulting Radiologist, Department of Radiology, Australia.

3. Associate Professor, Department of Radiology, Dr. D. Y. Patil Medical College, Pune, Maharashtra.

4. Senior Registrar, Department of Radiology, MGM Medical College \& Hospital, Aurangabad, Maharashtra.

\section{NAME ADDRESS EMAIL ID OF THE CORRESPONDING AUTHOR:}

Dr. Abhang Apte,

F41, Venus Row Houses, Behind Nishant Park Hotel, Beed Bypass Road, Aurangabad, Maharashtra, India. E-mail: drabhang@rediffmail.com

Date of Submission: 31/01/2014. Date of Peer Review: 01/02/2014. Date of Acceptance: 11/02/2014. Date of Publishing: 06/03/2014. 